\title{
Адсорбция диклофенака на MIL-96 и MIL-100 из нейтральных водных растворов: моделирование процесса адсорбции
}

\author{
Швыдко А.В. ${ }^{1}$, Тимофеева М.Н. ${ }^{1,2}$, Симонов П.А. ${ }^{2}$ \\ ${ }^{1}$ Новосибирский государственный технический университет, Новосибирск \\ ${ }^{2}$ Институт катализа им. Г.К. Борескова СО РАН, Новосибирск
}

Поступила в редакцию 9.10 .2020 г.

DOI: $10.17308 /$ sorpchrom.2021.21/3218

В последнее годы значительно возросло потребление диклофенака - нестероидного противовоспалительного средства с сильным обезболивающим и жаропонижающим действиями. Однако он является антропогенным загрязнителем из-за своей высокой стойкости к воздействию биологических систем очистки. В последнее время в области очистки сточных вод от различных органических загрязнителей, в том числе диклофенака, активно развиваются адсорбционные методы, поскольку они позволяют снизить остаточные концентрации вещества в растворе до уровня чувствительности физических методов

В работе изучена адсорбция диклофенака из нейтральных водных растворов с использованием в качестве адсорбентов металлорганических координационных полимеров, MIL-96 и MIL-100, образованных ионами алюминия (III) и 1,3,5-бензолтрикарбоновой кислотой. Адсорбция была изучена при $20^{\circ} \mathrm{C}$ из растворов с $\mathrm{pH}=6.0-6.2$, начальной концентрацией ДКФ 40-800 мг/дм ${ }^{3}$ и концентрацией адсорбента $1 \mathrm{mг} / 4 \mathrm{~cm}^{3}$. Показана высокая адсорбционная ёмкость 473-541 мг/г изученных МОКП, позволяющая снизить остаточную концентрацию диклофенака натрия в растворе до уровня чувствительности УФ-спектроскопии. Максимальная величина адсорбции диклофенака на MIL-96 и MIL-100 равна 475 и $480 \mathrm{Mг} / г$, соответственно.

Bce адсорбционные изотермы были L-типа. Экспериментальные изотермы адсорбции были проанализированы с помощью моделей Ленгмюра, Фрейндлиха и Темкина для описания распределения молекул диклофенака между адсорбентом и жидкостью. Были определены константы и параметры адсорбционных моделей. Полученные данные указывали на то, что адсорбция диклофенака протекает по механизму физической сорбции. Согласно модели Фрейндлиха поверхность MIL-96 имеет энергетически неэквивалентные адсорбционные центры по сравнению с MIL-100. Высокая микропористость MIL-96 по сравнению с MIL-100 способствовала протеканию на поверхности MIL-96 полислойной адсорбции диклофенака. Путем сопоставления среднеквадратичных отклонений $\left(\mathrm{R}^{2}\right)$, сделан вывод о том, что модель Фрейндлиха лучше других описывает адсорбцию диклофенака на MIL-96, а модель Ленгмюра - адсорбцию диклофенака на MIL-100. Этот вывод так же согласовывался с различием их структурных и текстурных свойств.

Ключевые слова: адсорбция, диклофенак, металлорганические каркасы, MIL-100, MIL-96, сточные воды.

\section{Введение}

Бурное развитие фармацевтической промышленности, а также неконтролируемое поступление лекарственных препаратов в окружающую среду оказывает негативное влияние на человека и окружающую среду. Многочисленные исследования по очистке водных объектов показали, что лекарственные препараты удаляются на очистных сооружениях частично, степень очистки не превышает 
90-95 \%. К тому же они не поддаются биологическому разложению.

Одним из наиболее стойких к воздействию обычных биологических систем очистки является диклофенак (ДКФ) (рис. 1) - лекарственное средство, производное фенилуксусной кислоты, относящееся к фармакологической группе нестероидных противовоспалительных препаратов, с сильным обезболивающим, жаропонижающим и противовоспалительным действиями [1]. Неконтролируемое поступление ДКФ в окружающую среду оказывает негативное влияние на человека, увеличивая риск возникновения язвы желудка, заболеваний печени и др., а также приводит к возникновению лекарственной устойчивости патогенных микроорганизмов по отношению к антибактериальным средствам.<smiles>COC(=O)Cc1ccccc1Nc1c(Cl)cccc1Cl</smiles>

Рис. 1. Структура диклофенака

Fig. 1. The structure of diclofenac

В настоящее время интенсивно разрабатываются адсорбционные методы очистки сточных вод от различных органических загрязнителей, в том числе ДКФ. Из всех известных методов (биологическая очистка, флотация, коагуляция, фотоокисление, электрохимическое окисление) адсорбционные методы являются наиболее эффективными, поскольку они в некоторых случаях позволяют удалять до 100\% загрязняющего вещества. Для удаления ДКФ из водной среды методом адсорбции в качестве адсорбентов предложено использовать активные угли [2-5], алюмосиликаты (цеолиты, силикагели, глины и др.) [6-7], модифицированные полимерные материалы [8-9] и др. Их высокая адсорбционная емкость (300900 мг/г) наблюдается в кислых средах, то есть требует дополнительного введения в систему кислоты.
Недавно Жуанг и соавт. [10] показали возможность удаления ДКФ из нейтральных водных растворов (рН 6.0-6.2) с использованием металлорга-нического координационного полимера (MOKП) MIL$100(\mathrm{Fe})$. Его максимальная адсорбционная емкость достигала 778 мг/г, что сопоставимо с величинами адсорбции, характерными для углеродных адсорбентов.

Поскольку поиск эффективных сорбционных материалов для очистки сточных вод от диклофенака является актуальной задачей, целью данной работы было исследование сорбционных возможностей из нейтральных водных растворов ( $\mathrm{pH}$ 6.0-6.2) Al-содержащих МОКП, таких как микро/мезопористый MIL-100 и микропористый MIL-96, по отношению к ДКФ, а также исследование механизма адсорбции для дальнейшего их использования в качестве сорбентов в процессах водоочистки. Для достижения поставленной цели решались следующие задачи:

- изучение процесса адсорбции диклофенака на MIL-100 и MIL-96 в статических условиях;

- изучение механизма адсорбции с использованием адсорбционных моделей Ленгмюра, Фрейндлиха и Темкина.

\section{Экспериментальная часть}

В работе использовали диклофенак натрия (Aldrich), гидроксид натрия «ч.д.а», нитрат алюминия $\left(\mathrm{Al}\left(\mathrm{NO}_{3}\right)_{3} \cdot 9 \mathrm{H}_{2} \mathrm{O}\right)$ (Aldrich, 98\%), тримезиновую кислоту (1,3,5-бензолтрикарбоновая кислота, $\mathrm{H}_{3}$ ВТС) (Aldrich, 98\%), 1,3,5триметилбензо-ловую кислоту (МезBTC) (Aldrich, $98 \%$ ). Металлорганические координа-ционные полимеры MIL96 и MIL-100 были синтезированы гидротермальным способом согласно методике, описанной в работе [11]. Текстурные характеристики образцов приведены в табл. 1.

Адсорбционные измерения проводили во встряхиваемой стеклянной ампуле объёмом $5 \mathrm{~cm}^{3}$. В ампулу загружали $1 \mathrm{~cm}^{3}$ 
Таблица 1. Текстурные свойства Al-МОКП

Table 1. Texture properties of MIL-96 and MIL-100

\begin{tabular}{|l|c|c|c|}
\hline \multicolumn{1}{|c|}{ MOКП } & $\mathrm{S}_{\mathrm{BET}, \mathrm{M}^{2} / \Gamma}$ & $\mathrm{V}_{\Sigma}, \mathrm{cm}^{3} / \Gamma$ & $\mathrm{V}_{\mu}, \mathrm{cm}^{3} / \Gamma$ \\
\hline MIL-96 & 315 & 0.14 & 0.13 \\
\hline MIL-100 & 1249 & 0.73 & 0.30 \\
\hline
\end{tabular}

взвеси, содержащей 10 мг МОКП в $10 \mathrm{~cm}^{3}$ воды. Через определённые промежутки времени отбирали пробы раствора. МОКП отделяли центрифугированием. После разбавления раствора в 10 раз $0.1 \mathrm{M}$ раствором гидроксида натрия измеряли концентрацию ДКФ спектрофотометрически (200-600 нм). УФ-спектр снимали на спектрофото-метре Cary-50 Scan (толщина слоя

10 мм, длина волны - 277 нм). Количество адсорбированного ДКФ рассчитывали по формуле (1):

$$
q=\frac{\left(C_{0}-C_{p}\right) \cdot V_{д K \Phi}}{m_{\text {адс }}},
$$

где $q$ - величина адсорбции, мг/г; $C_{0}$ - начальная концентрация ДКФ, мг/дм ${ }^{3} ; C_{p}$ - равновесная концентрация

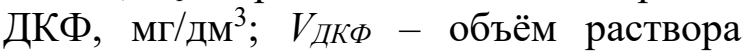
ДКФ, дм $;$ тадс - масса адсорбента, $\mathrm{Mг} / \mathrm{cm}^{3}$. Погрешность измерений составила $\pm 1.8 \%$.

\section{Обсуждение результатов}

Описание и моделирование экспериментальных данных. Изотермы адсорбции ДКФ на MIL-96 и MIL-100, показаны на рис. 2. Согласно классификации Чарльза Гильса они относятся к классу L (изотермы Ленгмюра), то есть величина адсорбции $(q)$ возрастает с увеличением равновесной концентрации $\left(\mathrm{C}_{\mathrm{p}}\right)$ и постепенно приближается к максимальной

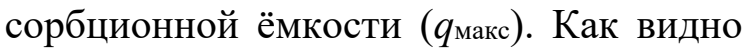
из данных рис. 2 максимальные величины адсорбции ( $\left.q_{\text {макс }}\right)$ равны 475 и 480 мг/г для MIL-96 и MIL-100, соответственно.

Для описания распределения молекул ДКФ между адсорбентом и жидкостью экспериментальные изотермы адсорбции были проанализированы с помощью моделей Ленгмюра, Фрейндлиха и Темкина [12-14].

\section{Модель Ленгмюра}

Модель Ленгмюра описывает гомогенный мономолекулярный процесс адсорбции, т.е. равномерное распределение адсорбированных молекул за счёт сил межмолекулярного взаимодействия на поверхности твёрдого тела, содержащего конечное число активных центров с равной энергией (локализованная адсорбция).

Линейная форма уравнения изотермы Ленгмюра описывается уравнением (2):

$$
\frac{1}{q}=\frac{1}{C_{p} K_{L}} \cdot \frac{1}{q_{\text {макс }}}+\frac{1}{q_{\text {макс }}},
$$

где $q$ - величина адсорбции, мг/г или

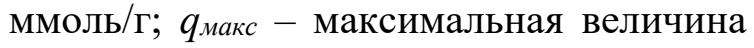

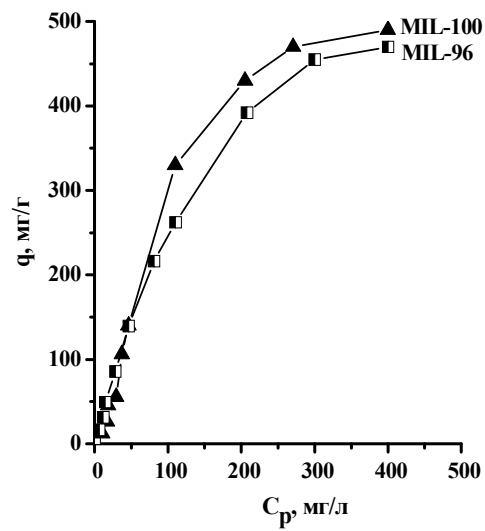

Рис. 2. Изотермы адсорбции ДКФ на Al-МОКП

Fig. 2. Adsorption isotherms of DCF on MIL-96 and MIL-100 
Таблица 2. Основные параметры моделей изотерм адсорбции ДКФ на Al-МОКП

Table 2. The calculated parameters from the Langmuir, Freundlich and Temkin adsorption isotherm models for MIL-96 and MIL-100

\begin{tabular}{|c|c|c|c|}
\hline \multirow{2}{*}{ Модель } & \multirow{2}{*}{ Параметры модели } & \multicolumn{2}{|c|}{ МОКП } \\
\hline & & MIL-96 & MIL-100 \\
\hline \multirow{5}{*}{ Модель Ленгмюра } & $q_{\text {экс }}, \mathrm{M} / \Gamma$ & 475 & 480 \\
\hline & $q_{\text {макс }}, \mathrm{M \Gamma} / \Gamma$ & 621 & 532 \\
\hline & $\mathrm{K}_{\mathrm{L}} 10^{3}$, дм$^{3} /$ ммоль & 5.55 & 4.30 \\
\hline & $\mathrm{R}_{\mathrm{L}}\left(\mathrm{C}_{\mathrm{o}}=150 \mathrm{мг} / \Gamma\right)$ & 0.548 & 0.606 \\
\hline & $\mathrm{R}^{2}$ & 0.974 & 0.972 \\
\hline \multirow{3}{*}{ Модель Фрейндлиха } & $\mathrm{K}_{\mathrm{F}}, \mathrm{M \Gamma} / \Gamma$ & 4.25 & 3.06 \\
\hline & $1 / \mathrm{n}$ & 0.861 & 0.911 \\
\hline & $\mathrm{R}^{2}$ & 0.977 & 0.920 \\
\hline \multirow{3}{*}{ Модель Темкина } & $\mathrm{K}_{\mathrm{T}}, \not \mathrm{M}^{3} / \mathrm{M \Gamma}$ & 0.055 & 0.069 \\
\hline & $\mathrm{b}$, Дж/моль & 15.8 & 16.2 \\
\hline & $\mathrm{R}^{2}$ & 0.968 & 0.963 \\
\hline
\end{tabular}

адсорбции, мг/г или моль/дм ${ }^{3} ; K_{L}-$ константа Ленгмюра, связанная с энергией адсорбции, дм 3 мммоль; $C_{p}$ - равновесная концентрация, мг/дм ${ }^{3}$ или ммоль/дм³ . Основные параметры, рассчитанные по данной модели, приведены в табл. 2. Линейные корреляции изотерм Ленгмюра показанны на рис. 3.

Для описания процесса адсорбции используют безразмерный параметр $\mathrm{R}_{\mathrm{L}}$, называемый коэффициентом разделения или параметром равновесия, который определяется по формуле (3):

$$
R_{L}=\frac{1}{1+K_{L} C_{0}},
$$

где $R_{L}$ - коэффициент разделения; $K_{L}$ - константа Ленгмюра, связанная с энергией адсорбции, дм ${ }^{3} /$ ммоль; $C_{0}-$ начальная концентрация, мг/дм ${ }^{3}$ или

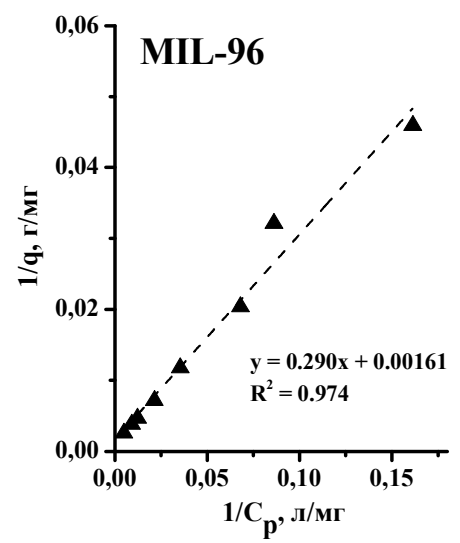

моль/дм³ ${ }^{3}$ Значение $\mathrm{R}_{\mathrm{L}}$ указывает на характер протекания адсорбции: неблагоприятная $\left(\mathrm{R}_{\mathrm{L}}>1\right)$, линейная $\left(\mathrm{R}_{\mathrm{L}}=1\right)$ или благоприятная $\left(\mathrm{R}_{\mathrm{L}}<1\right)$. Для MIL-96 и MIL-100 величины $\mathrm{R}_{\mathrm{L}}$, рассчитанные при начальной концентрации ДКФ 150 мг/дм ${ }^{3}$, равны 0.548 и 0.606 (табл. 2), соответственно, то есть находятся в диапазоне $0 \div 1$. Это указывает на благоприятную адсорбцию ДКФ на адсорбенте.

\section{Модель Фрейндлиха}

Модель Фрейндлиха описывает многослойную адсорбцию на гетерогенной поверхности, которая имеет энергетически неэквивалентные адсорбционные центры с экспоненциальным распределением величины энергии, то есть первоначально заполняются активные центры с максимальной энергией, а затем все остальные.

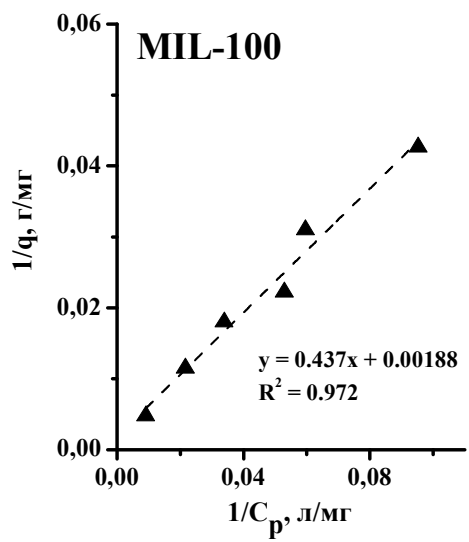

Рис. 3. Линейные корреляции изотерм Ленгмюра

Fig. 3. Linear correlations of Langmuir isotherms 

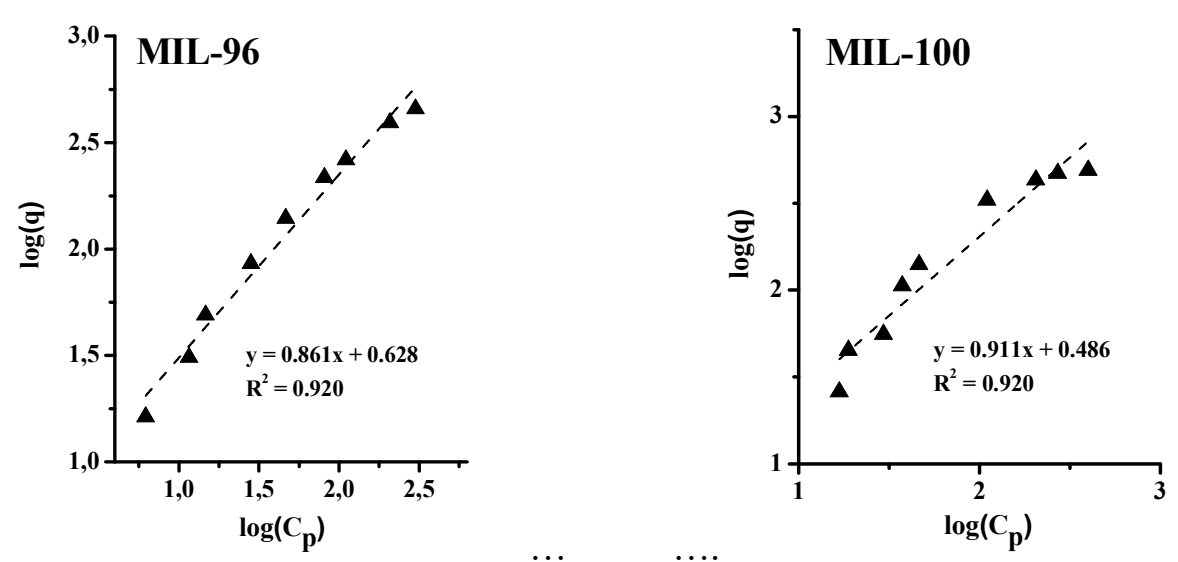

Рис. 4. Линейные корреляции изотерм Фрейндлиха

Fig. 4. Linear correlations of Freundlich isotherms

Линейная форма уравнения изотермы Фрейндлиха описывается уравнением (4):

$$
\log q=\log K_{F}+\frac{1}{n} \log C_{p},
$$

где $q$ - величина адсорбции, мг/г или ммоль $/ \Gamma ; K_{F}-$ константа Фрейндлиха (при

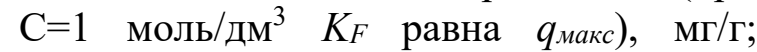
$C_{p}$ - равновесная концентрация адсорбтива в растворе, мг/дм ${ }^{3}$ или моль/дм 3 ; $1 / n$ - константа (адсорбционный показатель, зависящий от температуры и природы адсорбента). На рис. 4 приведены линейные корреляции Фрейндлиха для изотерм адсорбции ДКФ на MIL-96 и MIL-100.

Для описания применимости модели используют параметр $1 / \mathrm{n}$, который является показателем силы и характером протекания адсорбции: при 1/n равном 1 распределение адсорбированных частиц между двумя фазами не зависит от концентрации; при $1 / \mathrm{n}$ равном 0 адсорбция носит необратимый характер; при $1 / \mathrm{n}<1$

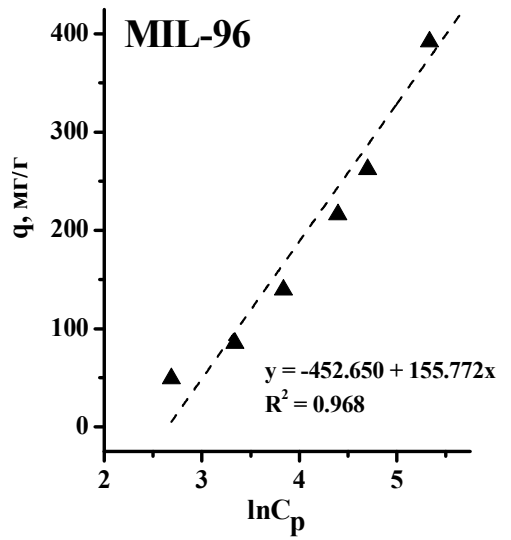

адсорбция благоприятная, а при 1/n>1 адсорбция неблагоприятная. Кроме того, параметр $1 / \mathrm{n}$ является показателем неоднородности адсорбента: чем он меньше, тем больше ожидаемая неоднородность. Как видно из данных табл. 2, величина 1/n для MIL-96 (0.861) меньше по сравнению с MIL-100 (0.911). Следовательно, можно предположить, что поверхность MIL-96 более неоднородна по сравнению c MIL-100.

\section{Модель Темкина}

Модель Темкина описывает адсорбцию частиц в монослое с учётом межмолекулярного взаимодействия «адсорбентадсорбат». Кроме того, эта модель характеризует равномерное распределение энергии связи (вплоть до некоторой максимальной величины), которое учитывает взаимодействие адсорбционных центров и частиц адсорбата.

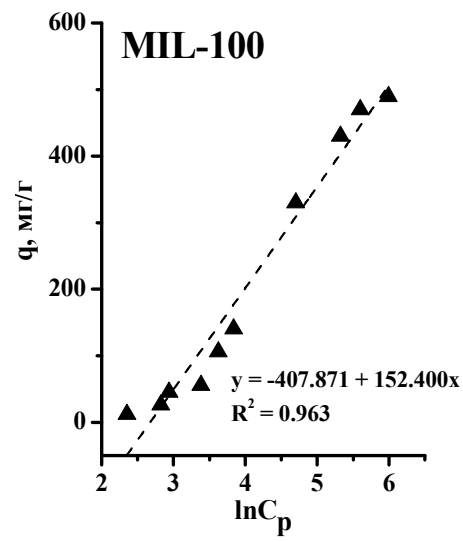

Рис. 5. Линейная корреляция изотермы Темкина

Fig. 5. Linear correlation of Temkin isotherm 
Линейная модель изотермы Темкина описывается уравнением (5):

$$
q=\frac{R T}{b} \ln K_{T}+\frac{R T}{b} \ln C_{p},
$$

где $q$ - величина адсорбции, мг/г или моль/г; $R$ - универсальная газовая постоянная; $T$ - температура, $K$; $b$ - константа, характеризующая теплоту адсорбции, Дж/моль; $K_{T}$ - константа Темкина или константа связывания при равновесии (константа сорбционного равновесия, соответствующая максимальной энергии связывания), дм ${ }^{3} /$ мг или дм $33 /$ моль; $C_{p}-$ равновесная концентрация, мгдм ${ }^{3}$ или ммоль/дм ${ }^{3}$. Как видно из рис. 5 экспериментальные изотермы адсорбции для MIL-96 и MIL-100 хорошо описываются моделью Темкина.

Характер адсорбции на MIL-100 и MIL-96 можно определить, основываясь на величине энергии адсорбционного взаимодействия между молекулами твёрдого тела и адсорбата (параметр $b$ ). Для физической адсорбции характерно присутствие Ван-дер-Ваальсовых сил, теплота адсорбции которых мала и не превышает 10-30 кДж/моль. В тоже время величина энергии химической адсорбции достигает несколько сотен кДж/моль. Рассчитанные значения параметра $b$ указывают на физическую адсорбцию ДКФ (табл. 2).

\section{Анализ полученных результатов}

Линейные формы изотерм Ленгмюра, Фрейндлиха и Темкина (ур. (2), (4) и (5)), показанные на рис. 3-5, имеют минимальные отклонения между экспериментальными данными адсорбции и теоретически вычисленными. Сравнивая данные, показанные в табл. 2, видно, что для описания адсорбции ДКФ на MIL-96 лучше всего подходит модель Фрейндлиха $\left(\mathrm{R}^{2}=0.977\right)$, в то время как для описания адсорбции MIL-100 - модель Ленгмюра $\left(\mathrm{R}^{2}=0.974\right)$. Это различие указывает на наличие неоднородных центров на поверхности MIL-96. Надо отметить, что адсорбционная емкость MIL-96 (475 мг/г) близка к емкости MIL-100 (480 мг/г), не- смотря на её низкую удельную поверхность (табл. 1). Это согласуется с различиями констант адсорбционного равновесия уравнений Ленгмюра и Фрейндлиха (табл. 2). Как видно, константы $\mathrm{K}_{\mathrm{L}}$ и $\mathrm{K}_{\mathrm{F}}$ для MIL-96 больше по сравнению с MIL100 , что указывает на большее сродство ДКФ к поверхности MIL-96. Это можно объяснить различием их структур. Структура MIL-96 представляет собой сотовую решетку с 18-членными кольцами, образующими изолированные трехъядерные

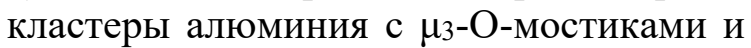
бесконечные цепочки октаэдров алюминия [11]. MIL-96 имеет три типа полостей с диаметром окон 2.5-3.5 А. В отличие от MIL-96 и MIL-100 представляет собой трехмерный каркас, имеющий два типа полостей с мезопора размером 25 и $29 \AA$, которые доступны через окна с диаметрами 5.5 и $8.6 \AA$. окна соответственно [15]. Основываясь на размере молекулы ДКФ $(5.2 \AA \times 7.4 \AA \times 10.3 \AA)$ видно, что её размер больше диаметра окон в MIL-96. Следовательно, адсорбция ДКФ протекает на поверхности в отличие от MIL-100.

Другое объяснение близостью величин адсорбции может быть связано с различием текстурных свойств MIL-96 и MIL-100 (табл. 1). Это предположение вытекает из сравнения отношения объема адсорбированного ДКФ к общему объёму пор МОКП, рассчитанного по формуле (6):

$$
R_{V}=\frac{V_{\text {дКФ }} \cdot q \cdot N_{A}}{V_{\Sigma}}
$$

где $R V$ - параметр, характеризующий объём адсорбированного ДКФ к общему объёму пор МОКП; $V$ - суммарный объем

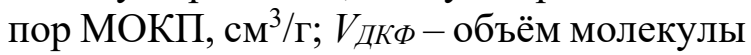
ДКФ $\left(5.2 \cdot 10^{-8} \mathrm{~cm} \times 7.4 \cdot 10^{-8} \mathrm{~cm} \times 10.3 \cdot 10^{-8} \mathrm{~cm}\right)$; $q$ - адсорбционная ёмкость МОКП, моль/г; $N_{A}-$ постоянная Авогадро; $V_{\Sigma}-$ общий объём пор, $\mathrm{cm}^{3} / \Gamma$. Рассчитанные значения Rv для MIL-96 и MIL-100 были 2.29 и 0.49 , соответственно. Из этих данных видно, что в отличие от MIL-100, в случае MIL-96 общий объём пор ниже, 
чем объём адсорбированных молекул ДКФ. Это указывает на адсорбцию не только в порах, но и на поверхности MIL-96.

В целом, выполненные исследования позволили установить адсорбционные характеристики MIL-96 и MIL-100 по отношению к диклофенаку как одному из типичных загрязнителей фармацефтического производства. Полученные величины адсорбции для MIL-96 (475 мг/г) и MIL-100 (480 мг/г) сопоставимы с величинами адсорбции, характерными для адсорбентов на основе углерода. Таким образом, полученные в работе результаты указывают на большие перспективы применения МОКП в качестве адсорбентов.

\section{Заключение}

Изучена адсорбция ДКФ из нейтральных водных растворов на МОКП, образованных ионами алюминия (III) и 1,3,5- бензолтрикарбоновой кислотой - MIL100 и MIL-96. Показана высокая адсорбционная ёмкость 475-480 мг/Г изученных Al-МОКП, позволяющая снизить остаточные концентрации в растворе до уровня чувствительности УФ-спектроскопии.

Экспериментальные изотермы адсорбции ДКФ на Al-MОКП описаны моделями Ленгмюра, Фрейндлиха и Темкина. Определены константы и параметры адсорбционных моделей. Показано, что адсорбция протекает по механизму физической сорбции. Поверхность MIL-96 имеет энергетически неэквивалентные адсорбционные центры по сравнению с MIL100. Показано, что адсорбция ДКФ на MIL-96 лучше всего описывается моделью Фрейндлиха В то же время процесс адсорбции диклофенака на MIL-100 наиболее точно описывается моделью Ленгмюра, что связано с различием их структурных и текстурных свойств

\section{Работа выполнена при поддержке РАН и ФАНО России (проект АAAA-A17-117041710082-8)}

\section{Список литературы}

1. Lonappan L., Brar S.K., Das R.K., Verma M. et al. // Environ. Intern. 2016. Vol. 96. pp. 127138.

2. Bhadra B.N., Ahmed I., Kim S., Jhung S.H. // Chem. Eng. J. 2017. Vol. 314, pp. 50-58.

3. Nam S.-W., Jung C., Li H., Yu M. et al. // Chemosphere. 2015. Vol. 136, pp. 20-26.

4. Mao N., Huang L., Shuai Q. // ACS Omega. 2019. Vol. 4, pp. 15051-15060.

5. Rigobello E.S., Dantas A.D.B., Di Bernando L., Vieira E.M. // Chemosphere. 2013. Vol. 92. pp. 184-191.

6. Abbasi Z., Shamsaei E., Leong S.K., Ladewig B. et al. // Micropor. Mesopor. Mater. 2016. Vol. 236. pp. 28-37.

7. Barczak M., Wierzbicka M., Borowski P. // Micropor Mesopor Mater. 2018. Vol. 264. pp. 254-264.

8. Janicijevic J., Krajisnik D., Calija B., Vasiljevic B.N. et al. // Int. J. Pharm. 2015. Vol.
496. pp. 466-474.

9. Lu X., Shao Y., Gao N., Chen J. et al. // Chemosphere. 2016. Vol. 161. pp. 400-411.

10. Dai C.M., Geissen S.U., Zhang Y.L., Zhang Y.J. et al. // Environ. Pollut. 2011. Vol. 159. pp. 1660-1666.

11. Zhuang S., Liu Y., Wang J. // Environ. Pollution. 2019. Vol. 253. pp. 616-624.

12. Khan N.A., Lee J.S., Jeon J., Jun C.-H. et al. // Micropor. Mesopor. Mater. 2012. Vol. 152. pp. 235-239.

13. Dada A.O., Olalekan A.P., Olatunya A.M., Dada O. // IOSR J. Appl. Chem. 2012. Vol. 3. pp. 38-45.

14. Tempkin M.I., Pyzhev V. // Acta Physiochim. URSS. 1940. Vol. 12. pp. 217-222.

15. Ayawei N., Ebelegi A.N., Wankasi D. // J. Chem. 2017. Vol. 2017. pp. 1-11.

16. Haouas M., Volkringer C., Loiseau T., Ferey G. et al. // Chem. Eur. J. 2009. Vol. 15. pp. 3139-3146. 


\title{
Adsorption of diclofenac on MIL-96 and MIL-100: modeling of adsorption process
}

\author{
(C) 2021 Shvydko A.V. ${ }^{1}$, Timofeeva M.N. ${ }^{1,2}$, Simonov P.A. ${ }^{2}$ \\ ${ }^{I}$ Novosibirsk State Technical University, Novosibirsk, Russian Federation \\ ${ }^{2}$ Boreskov Institute of Catalysis SB RAS, Novosibirsk, Russian Federation
}

In recent years, the consumption of diclofenac, a non-steroidal anti-inflammatory drug with strong analgesic and antipyretic effects, has increased significantly. However, it is a man-made pollutant due to its high resistance to biological treatment systems. One of the actively developing methods for wastewater treatment for various organic pollutants, including diclofenac, are adsorption methods, allowing reducing the residual concentration of a substance in a solution to the level of sensitivity of physical methods.

The adsorption of diclofenac from neutral aqueous solutions using as adsorbents metal-organic frameworks (MOFs), MIL-96 and MIL-100, formed by aluminium (III) ions and 1,3,5-benzene tricarboxylic acid was investigated in this study. The adsorption was studied at $20^{\circ} \mathrm{C}$ from solutions with $\mathrm{pH}=6.0-6.2$. The initial concentration of DCF was 40-800 mg/l and an adsorbent concentration was $1 \mathrm{mg} / 4 \mathrm{ml}$. A high adsorption capacity of $473-541 \mathrm{mg} / \mathrm{g}$ of the studied metal-organic MOFs was shown. The high adsorption capacity of MOFs allowed reducing the residual concentration of sodium diclofenac in solution to the level of sensitivity of UV spectroscopy. The maximal adsorption values of diclofenac on MIL-96 and MIL-100 were found to be 475 and $480 \mathrm{mg} / \mathrm{g}$, respectively.

All adsorption isotherms were of L-type. Experimental adsorption isotherms were analysed using the Langmuir, Freundlich, and Temkin models to describe the distribution of diclofenac molecules between the adsorbent and liquid. The constants and parameters of adsorption models were determined. The obtained data indicated that the adsorption of diclofenac is proceeded by the mechanism of physical sorption. According to the Freundlich model, the surface of MIL-96 has energetically nonequivalent adsorption centres in comparison with MIL-100. The high microporosity of MIL-96 compared to MIL-100 is facilitated the diclofenac multilayer adsorption on the MIL-96 surface. By comparing the standard deviations $\left(\mathrm{R}^{2}\right)$, it was concluded that the Freundlich model is better than others at describing the adsorption of diclofenac on MIL-96, and Langmuir's model better describes the adsorption of diclofenac on MIL-100. This conclusion was also consistent with the difference in their structural and textural properties.

Keywords: adsorption, diclofenac, metal-organic frameworks, MIL-100, MIL-96, wastewater.

\section{References}

1. Lonappan L., Brar S.K., Das R.K., Verma M. et al., Environ. Intern., 2016, Vol. 96, pp. 127138.

2. Bhadra B.N., Ahmed I., Kim S., Jhung S.H., Chem. Eng. J., 2017, Vol. 314, pp. 50-58.

3. Nam S.-W., Jung C., Li H., Yu M. et al., Chemosphere, 2015, Vol. 136, pp. 20-26.

4. Mao N., Huang L., Shuai Q., ACS Omega, 2019, Vol. 4, pp. 15051-15060.

5. Rigobello E.S., Dantas A.D.B., Di Bernando L., Vieira E.M., Chemosphere, 2013, Vol. 92, pp. 184-191.

6. Abbasi Z., Shamsaei E., Leong S.K., Ladewig B. et al., Micropor. Mesopor. Mater., 2016, Vol. 236, pp. 28-37.

7. Barczak M., Wierzbicka M., Borowski P., Micropor Mesopor Mater., 2018, Vol. 264, pp. 254-264.

8. Janicijevic J., Krajisnik D., Calija B., Vasiljevic B.N. et al., Int. J. Pharm., 2015, Vol.
496, pp. 466-474.

9. Lu X., Shao Y., Gao N., Chen J. et al., Chemosphere, 2016, Vol. 161, pp. 400-411.

10 Dai C.M., Geissen S.U., Zhang Y.L., Zhang Y.J. et al., Environ. Pollut., 2011, Vol. 159, pp. 1660-1666.

11. Zhuang S., Liu Y., Wang J., Environ. Pollution, 2019, Vol. 253, pp. 616-624.

12. Khan N.A., Lee J.S., Jeon J., Jun C.-H., et al., Micropor. Mesopor. Mater., 2012, Vol. 152, pp. 235-239.

13. Dada A.O., Olalekan A.P., Olatunya A.M., Dada O., IOSR J. Appl. Chem., 2012, Vol. 3, pp. 38-45.

14. Tempkin M.I., Pyzhev V., Acta Physiochim. URSS, 1940,Vol. 12, pp. 217-222.

15. Ayawei N., Ebelegi A.N., Wankasi D., J. Chem., 2017, Vol. 2017, pp. 1-11.

16. Haouas M., Volkringer C., Loiseau T., Ferey G. et al., Chem. Eur. J., 2009, Vol. 15, pp. 3139-3146. 
Швыдко Алина Витальевна - магистрант кафедры инженерных проблемы экологии, Новосибирского государственного технического университета, Новосибирск

Тимофеева Мария Николаевна - д.Х.н., профессор кафедры инженерных проблемы экологии Новосибирского государственного технического университета, ведущий научный сотрудник Института катализа им. Г.К. Борескова СО РАН, Новосибирск

Симонов Павел Анатольевич - к.х.н., ведущий научный сотрудник Института катализа им. Г.К. Борескова СО РАН, Новосибирск
Shvydko Alina V. - Master Student, Department of Engineering Issues in Ecology, Novosibirsk State Technical University, Novosibirsk, E-mail: alina.schwydcko.98@mail.ru

Timofeeva Maria N. - Doctor of Chemical Science, Professor, Department of Engineering Issues in Ecology, Novosibirsk State Technical University; Leading Research Fellow of Boreskov Institute of Catalysis SB RAS, Novosibirsk, E-mail: timofeeva@catalysis.ru

Simonov Pavel A. - PhD in Chemical Sciences, Senior Researcher of Boreskov Institute of Catalysis SB RAS, Novosibirsk, E-mail: simonov@catalysis.ru 